\title{
Integrated IOT based design and Android operated Multi-purpose Field Surveillance Robot for Military Use
}

\author{
${ }^{1}$ M.Ashokkumar, ${ }^{2}$ Dr.T.Thirumurugan \\ Department of Electronics and Communication Engineering \\ Christ Institute of Technology \\ Pondicherry, India \\ ashok5june@gmail.com, thiru0809@gmail.com
}

\begin{abstract}
This project describes about the design, construction and fabrication of multi-purpose field surveillance robot that can be used for land mine detection, toxic gas sensing and temperature and humidity sensor monitoring in war fields without putting serious manual risks. The land mine detector can detect covered metals, gas sensor can detect toxic gas attacks and the robot can be controlled wirelessly by Android phone. The robot uses Arduino Uno microcontroller to gather sensor information and NodeMCU WiFi to interface the controller and the robot. Based on the input information from Android application, the robot can make moved and climbed on any terrains. The distinguishing feature of our project from traditional ones is that the integrated design of Android phone operation and multiple IoT cloud servers. All robotic sensor information are delivered to cloud servers and viewed through Webpage. This way the robot can be used both at Military war fields and monitored at Military headquarters simultaneously. This is a novel attempt to integrate field robots and IoT technologies at an expandable mode of design. Additional enhancement of the design made it an outstanding choice for deployment and use in dangerous zones infested with land mines and other hazardous metallic items.
\end{abstract}

Keywords- Robotics, Embedded Systems, Internet of Things (IoT), Wireless Communication and Cloud Technologies

\section{INTRODUCTION}

A landmine is an explosive device implanted in the earth and triggered by pressure, magnetic fields and tripwires, among several others. They are one of the most common weapons used in contemporary combats and are most often used as preemptive barriers and opponent deterrents. They are trifling, usually round devices intended to hurt or kill people by an explosive detonation or flying fragments. Most mines are made of plastics and contain about the large quantity of metal as the spring in a ballpoint pen. The growth of the anti-tank mine was encouraged by the use of battle tanks during World War I. Anti-personnel mines were established to replace these larger mines that could easily be removed by enemy soldiers. The development and use of the landmines we know today became a chief military strategy in the middle of 1918 and 1939. At this time, mines were castoff in a careful manner and precisely targeted at militaries. It wasn't until the 1960s that the unsystematic distribution of landmines began. Today, there are still tens of millions of anti-personnel mines in the ground and amassed in over 60 nations. Landmines have wounded and killed thousands of army men in many conflicts.

- Landmine fatalities deprive communities and families of salary earners and precious parents, relations, spouses, and relatives.

- $\quad$ Per United Nations, around 2,000 people are killed or injured by landmines every month.

- Over half the landmine fatalities are civilians. Among civilian losses, more than $40 \%$ are children.

- A mine costs between $\$ 3$ and $\$ 30$. The cost to neutralize a mine is between $\$ 300$ and $\$ 1,000$ and surgical care costs about $\$ 3,000$ per amputee in developing countries. Buried landmines can continue active for over 50 years.

- Landmines have battered and killed thousands of Army men in every battle since the 1900 s, including the recent wars in Iraq and Afghanistan.

- Landmines set in motion a series of events that can lead to environmental damage in the form of soil degradation, deforestation, and the pollution of water resources.

\section{A. Why Robots are used for Land mine detections?}

Robots can be engaged to complete work in hazardous regions and can be used to accomplish difficult unpredictability levels in such regions. Progressively robots are becoming enthusiastically vital for standard subject applications, for instance, Metropolitan Hunt and Salvage and Military applications. A diversity of small robotic applications is now arising and robots are utilized to complete an assortment of errands. By and large, robots are quiet employed for unsafe work which is dangerous for humans, e.g., controller robot, spy robot, salvage robot, therapeutic operation and so forth. The use of robots in warfare, although traditionally a topic for science fiction, is 
being researched as a possible future means of fighting wars. Military robots are autonomous robots or remotecontrolled mobile robots designed for military applications, from transport to search \& rescue and attack.

Landmine identifying robot is utilized to search for metal objects buried up in the ground. Army bomb -disposal experts use metal detectors to scan for area mines covered up beneath streets and in mine fields. Technicians also use metal detectors to scan for wire cables concealed in ramparts. At airport, metal finders are utilized to scan voyagers for metal complaints, for example, cuts and firearms. According to various reports, the number of robots used worldwide is constantly increasing. They are more and more present in different workplaces such as manufacturing, processing operations, dangerous areas, medical environments, military, inaccessible areas etc. Also, robots are able to do social works like assisting people with disabilities or even playing when toys are robotic techniques based

This project focuses on designing and developing a robotic vehicle that can sense metals in front of it on its way like detecting land mines. A metal detector circuit interfaced to the control unit that alarms the user about a suspected metal ahead. The metal detector circuit is mounted on a robotic vehicle and its operation is to detect metals underneath automatically.

\section{B. Metal Detector Basics and theory}

A magnetic field (Fig. 1.) is produced around a coil, because of passing electric current through it. Metal detectors deal with the principle of transmitting a magnetic field and dissecting a return signal from the objective and environment. The magnetic transmitter is a transmit coil that produce a varying electric current by transmit electronics. The receiver is a coil that is connected to receive and signal processing electronics. The coils are inside a coil housing and all the

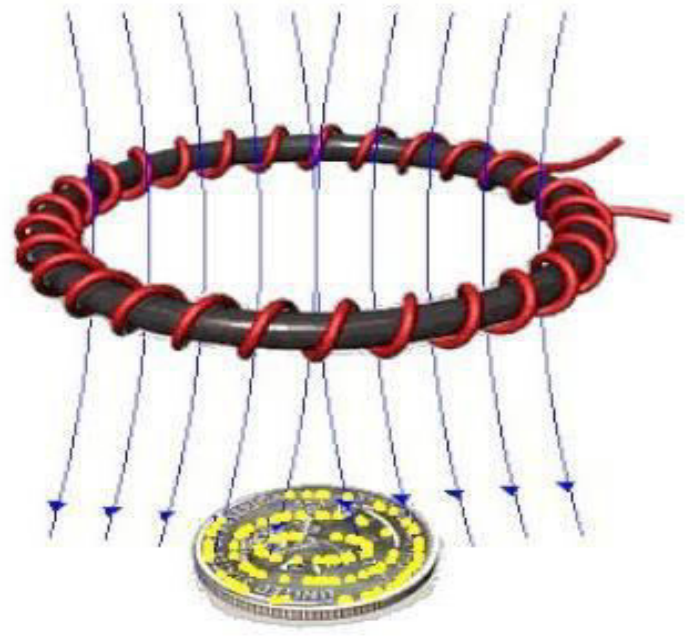

Figure 1. Magnetic field around the coil

hardware are within the electronics housing connected to the coil via an electric cable and usually called the "control box". A metal detector also includes an oscillator which delivers alternating current. At the point when this alternating current goes through the transmit coil present in a metallic object interacts with the coil, it produces another magnetic field around it. The transmitted magnetic field differs in time, generally at rates of genuinely sharp sound signals. This changing transmitted magnetic field flows electric currents in metal objects and they are called eddy currents. The metal detector has another coil in its loop called receiver coil. The receiver coil identifies the changes in the magnetic field that is caused due to presence of the metallic object. An electromagnetic field is a physical field produced by electrically charged metal objects. This refers to both the transmit field from the detector's search coil and the receive field from a target.

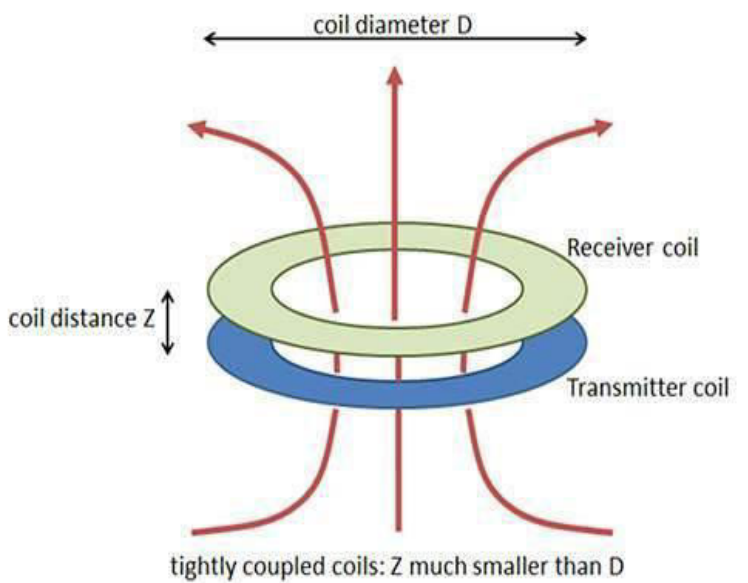

Figure 2. Transmitter and Receiver coils

The signal induced in the receiver coil can be considered as made up of two synchronous components:

- One component is same as the transmit signal that is called the reactive signal ("X").

- The other is called the resistive or loss component ("R"). It is the magnitude of the received signal.

The above mentioned both target $\mathrm{X}$ and $\mathrm{R}$ signals differ depending on the separation of the objective from the coil; the larger the distance, the weaker the transmitted magnetic field at the object and vice versa.

\section{Air Coil Inductors}

Before going further we need to understand concept of inductor and RLC circuits. Let us first discuss about inductors. Inductors are nothing but coils of enameled copper wire comes in different shapes and sizes. Based on various parameters the inductance of an inductor is calculated. In all of those parameters we are basically interested in core on the inductor as depending on the core, inductance value changes drastically 


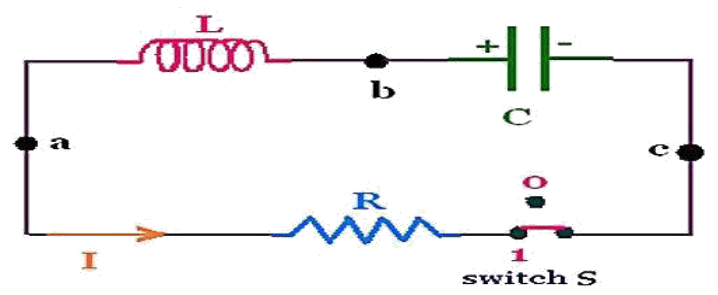

Figure 3. RLC Circuit

In the air inductors there will be no solid core. They are basically coils left in air. The medium of flow of magnetic field generated by inductor is nothing or air. These inductors have inductances of very less value. Now for a RLC circuit as shown in figure, the reactance or impedance between terminals " $\mathrm{a}$ " and "c" depends on the values of $\mathrm{L}$ and $\mathrm{C}$ if the applied signal frequency is constant. So if the inductance value changes the value of reactance or impedance changes. When a metal piece is brought near the coil, the metal piece acts as a core for the air cored inductor. By this metal acting as a core, the inductance of the coil changes or increases considerably. Thus making the signal to change resulting in variation to sound generated in speaker.

The inductance of a coil depends on four physical factors: (1) the number of turns, (2) the diameter of the form the coil is wound on, (3) the permeability of the core, and (4) the length of the coil. This provided by Wheeler's formula:

$$
L=\frac{0.8 \cdot a^{2} \cdot N^{2} \cdot \mu_{r}}{6 \cdot a+9 \cdot b+10 \cdot c} \llbracket i n, \mu H \rrbracket
$$

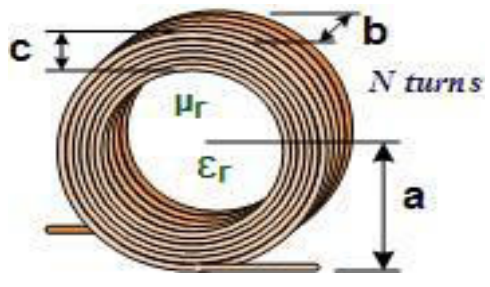

Figure 4. Multi-Layer Air-Core Inductance

\section{Integrating Robots into the Internet of Things}

The Internet of Things (IoT) organization allows connections between different entities (i.e., human beings, wireless sensors, mobile robots, etc.), using different but interoperable communication protocols and makes a dynamic multimodal/ heterogeneous system. In this setup, these diverse entities (observed as "things") have the capability to determine and discover one another, gather, provide or transmit information to IoT.

It is challenging to estimate the future evolution of Internet of Things (IoT) when it is estimated that the number of devices online by 2020 ranges from 50 billion to one trillion. Such potential can be exploited by robots. Still, in order to get there, new robots must address the need for unique identification and interoperability between them and other things from IoT, such as sensors, embedded devices, etc. Also, if robots use only the information provided by their own sensors, the applications will be limited. Unfortunately, many of current robots are not abundant enough to solve many multifaceted tasks - for example, concerning dynamic surroundings. But novel welfares can be attained over the application of innovative results from diverse exploration areas.

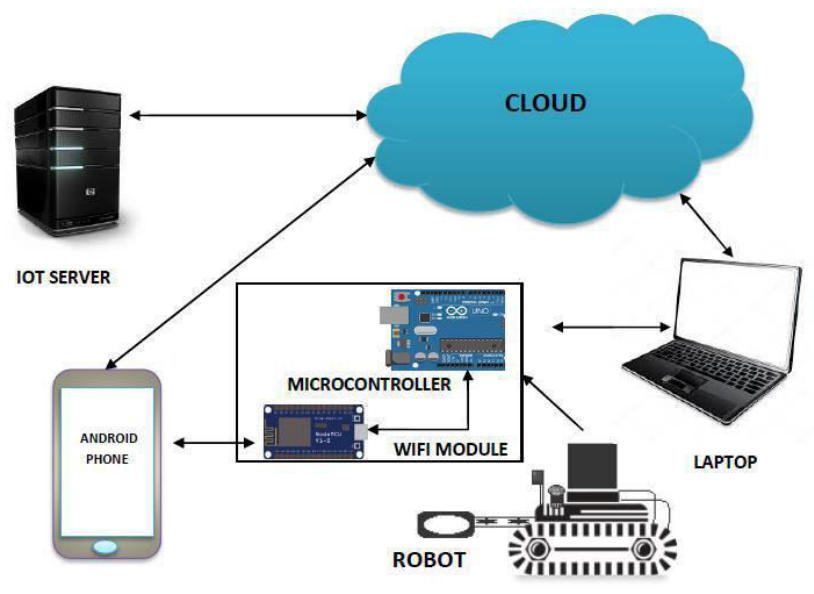

Figure 5. IoT Integration of Robots

Earlier work, they designed and developed a metal detector robotic vehicle using Android Bluetooth device that can be used in metal detection so the robot instead of humans. This has a limited range of operation i.e. $10 \mathrm{~m}$ from the hand held devices. Even while using WiFi module the data integrated was constrained only to Android App but not integrated to any other IoT platforms which is a poor design of model. Also inadequate amount of sensor on the robot carrying vehicle constrained the robot. Moreover the cost of device is more expensive in other existing model in the works. This project focuses on pioneer design to improve on the existing robot model in the world in an affordable and cost-effective way. This is a novel attempt to integrate wireless and IoT technologies on a same platform at an expandable mode of design. This design provide the robot a path breaking feature to connect to any number of IoT devices around the world and to be operated at anywhere and anytime using Internet Cloud technologies

\section{ROBOT CHARACTERISTICS AND THEIR COMPONENTS}

Robot platform is selected to be perfect for making robotic vehicle, smart robots and robotic projects which can be used for various applications and IoT projects. This is an excellent rover robotics platform for development of any autonomous robotic projects without spending a lot of money. Robotic platform consists of high quality, multi- 
purpose, laser cut acrylic double layer robotic chassis fitted with 2 DC Geared motors, 2 dummy motor shafts and 4 wheels. Double sided foam pieces and Holes are provided on acrylic platform to mount the circuitry, board or sensors easily. It can be installed with different electronic components, sensors, microprocessors and IoT devices.

\section{A. Robotic Vehicle Specifications}

Simple body structure moved on four-wheel driven by two motors using differential drive steering mechanism with powerful batteries. Durability and reliability test of the driving motors system, and shafts fitted with a bolts and nuts. It is remote reliable control and half automatic mode robot

.Using WiFi transceiver, and using handheld Android Phone to control the robot. The driving system and electronic circuits are feed from $12 \mathrm{~V}$ lead acid battery that can be recharged by DC power supply.Following are the characteristics of the robotic vehicle.

- Laser cut smooth chassis

- High Quality acrylic platform

- Low cost

- Mounting holes and foam pieces for easy mounting of the circuitry.

- Sufficient Space for mounting sensors, development boards, Batteries etc.

\section{B. Robot Dimensions}

The full length of the body including the metal sensor arm is $0.40 \mathrm{~m}$. The length of the body excluding the metal sensor arm is $0.26 \mathrm{~m}$. The width of vehicle is $0.22 \mathrm{~m}$ including the tire. The height of vehicle is $0.6 \mathrm{~m}$ from the ground and $0.16 \mathrm{~m}$ including the battery on the top of the vehicle. The full weight is less than $2 \mathrm{~kg}$ including the sensors.

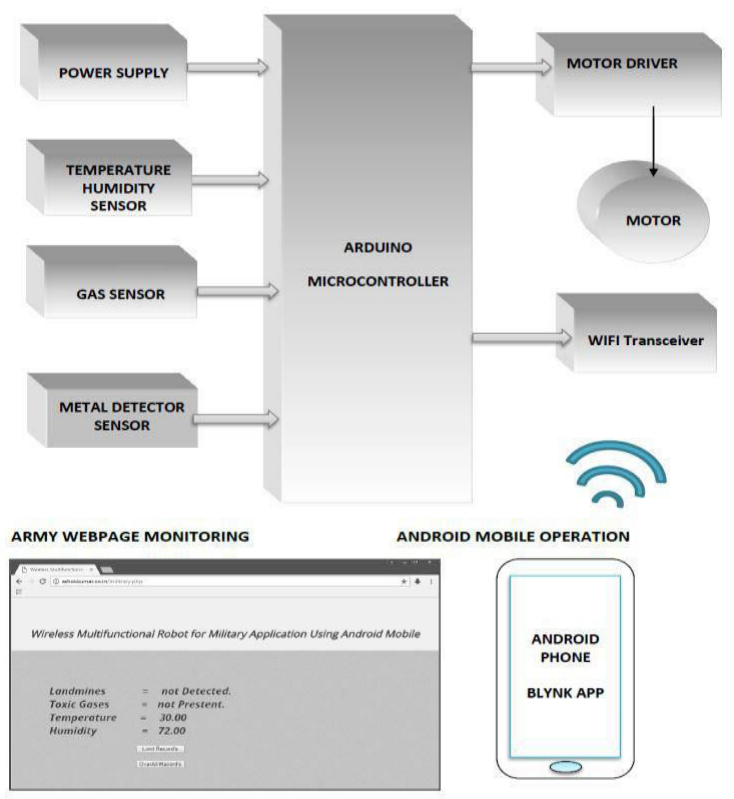

Figure 6. Overall Block Diagram of Robot Implementation

\section{Android Control \& Monitoring Section}

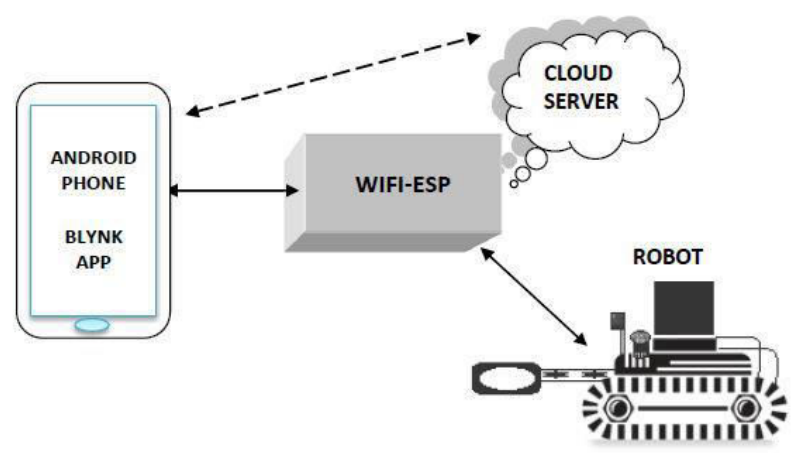

Figure 7. Android App Control and Monitoring section]

D. Web Software Section

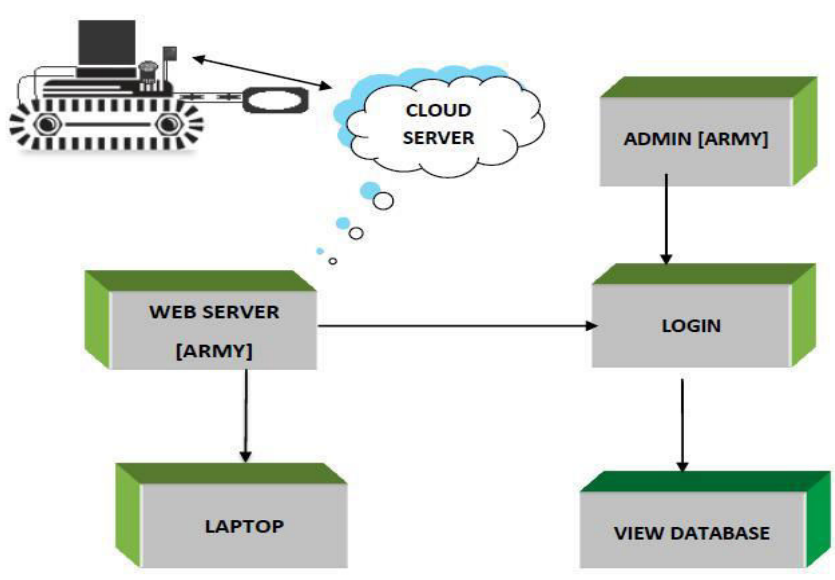

Figure 8. Website design and monitoring section

\section{HARDWARE ASSEMBLY \& SOFTWARE INTEGRATION}

An embedded system is a combination of computer hardware and software, either fixed in capability or programmable, that is designed for a specific function or for specific functions within a larger system. Robots are an application of Embedded Systems. Robots are constructed using hardware components like microcontrollers, motors, sensors, relays and integrated with sensible software programs using Embedded $\mathrm{C}$, which are designed to perform Industrial robotic machines, agricultural and process industry robotic devices, automobile robots, medical robots, and toy robots are robotic embedded systems. Nowadays, the Internet of Things (IoT) drives the robotic embedded systems base. This research study introduces the concept of the IoT where intelligent devices can monitor events, fuse sensor data from a variety of sources, use local and distributed "intelligence" to determine a best course of action and then act to control or manipulate objects the physical world and in some cases while physically moving through that world. 


\section{A. Hardware Design Framework}

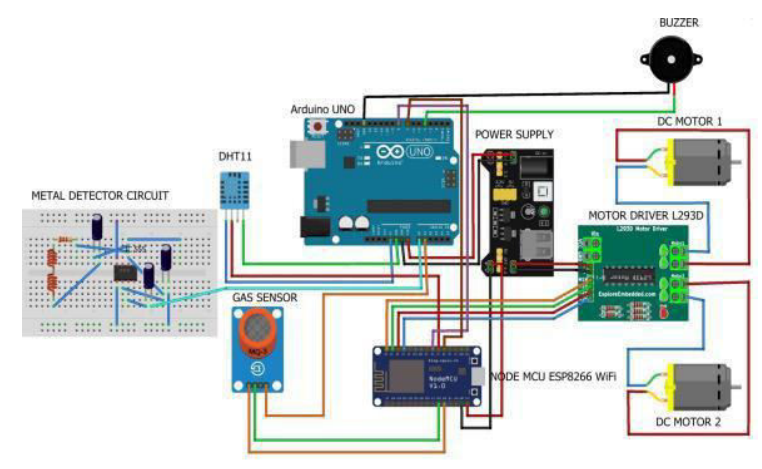

Figure 9. Robot Schematic Design Diagram

Robot Hardware is designed by Fritzing labs where each component is individually tested, hardware assembled and software integrated on the single robotic platform.

\section{B. Circuit Connections}

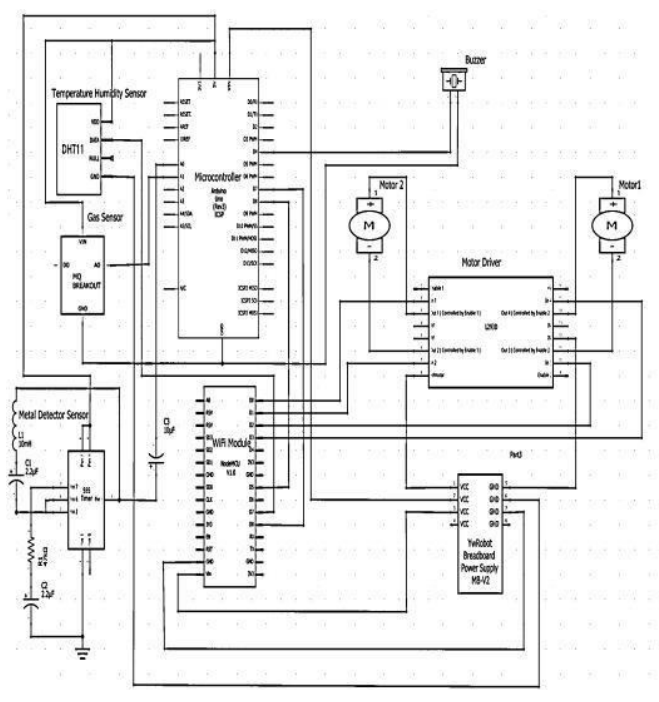

Figure 10. Robot Circuit Design Diagram

- The main units of robots are Arduino Uno microcontroller and NodeMCU WiFi device. They are connected to 5V DC power supply.

- Metal detection sensor and Gas sensor provide analog values when a metal or toxic gas is detected.

- Their output data pins are connected to analog pins A0, A1 of Arduino microcontroller.

- Once metal or toxic gas is detected, the sensor will send a high analog value to the A0 and A1 pins in the microcontroller.
- The microcontroller compares the high data signal to the threshold alcohol value. Once the analog value is greater than the threshold value, the microcontroller send a high data signal to Digital Pins D6 and D8 digital pins

- Digital pins D6 and D8 of microcontroller are connected to same kind of digital pins D6 and D8 of the WiFi module.

- Temperature and Humidity sensor DHT11 is connected directly to D7 digital pin WiFi module since it provide the data accurately.

- Digital pins D0, D1,D2 and D3 are input pins from WiFi module to two motor inputs $1 \& 2$ of Motor Driver L293D.

- Digital pins D0, D1, D2 and D3get the inputs from the Blynk Application which will be used to drive the two motors.

- Two DC gear motors are connected to two output pins of L293D.

\section{RESULTS}

\section{A. Robot Vehicle Fabricated}

Once the hardware components are assembled on the robot carrying vehicle, the software was integrated into the Arduino microcontroller and WiFi module of the robot components. The sensor systems were mounted on the carrying vehicle and the power supply was switched on the robot. 4G data was enabled on Android phone and the WiFi hotspot was turned on to facilitate the WiFi module of robot to get connected with the Internet Cloud platform. This is the best way for IoT integration for any robots in the world. Immediately the robot connected to the Internet Cloud platform using WiFi hotspot of android phone. The WiFi module of robot was displayed as WiFi hotspot user in the Tethering section of WiFi settings. This confirmed the online coming of the WiFi robot and connectivity to outside world of Cloud technologies.

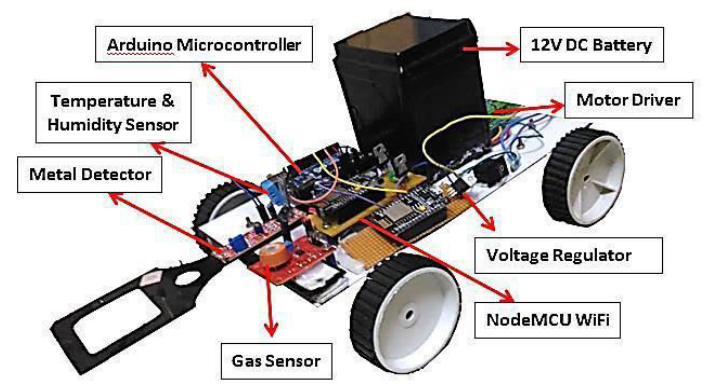

Figure 11. Multi-purpose Field Surveillance Robot Vehicle

Following are the instruction steps to control the robot once the robot comes online through the WiFi receiver and the Android phone: 
- User installed the Blynk App from Android Store on Android Phone. User created a Blynk account.

- User logged into the application and clicked on the 'Field Surveillance Robot' project.

- User clicked on the robot 'Go Live' button to connect the robot.

- User clicked on the 'Forward' and 'Backward' buttons to move and control the robotic vehicle.

- Robot successfully detected suspicious metal objects and alarmed the user nearby with buzzer sound. User received an alert from the robot in the Android Phone.

Robot successfully gathered the temperature and humidity information of the area under surveillance and sent the information back to the Android Phone. Thus the robot can able to detect the metals, toxic gases and monitor temperature humidity of the field using the sensors installed on the carrying vehicle. In order to achieve the accuracy in the detection and monitoring process, User conduct fine tune the sensor meters to increase accuracy and efficiency on the system. The voltage levels are regulated to desired operating range of the vehicle and micro controllers. Henceforth the robot is a novel design framework of modern IoT robots in the field.

\section{B. Test Case Scenario}

Following are the testing use cases of multi-purpose field surveillance robot below:

\section{MOTOR DRIVER VERIFICATION}

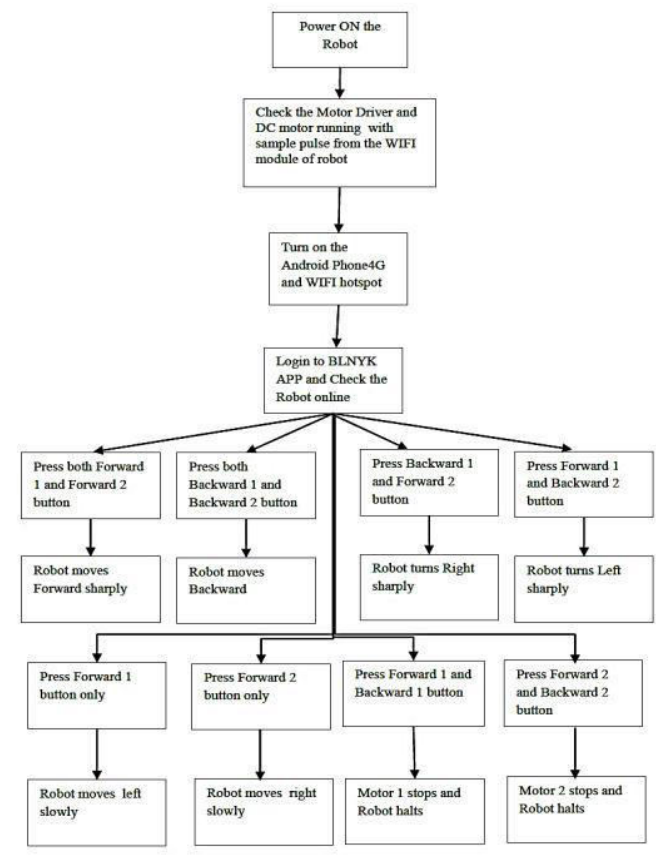

Figure 12. Motor Verification Flowchart

\section{METAL DETECTION VERIFICATION}

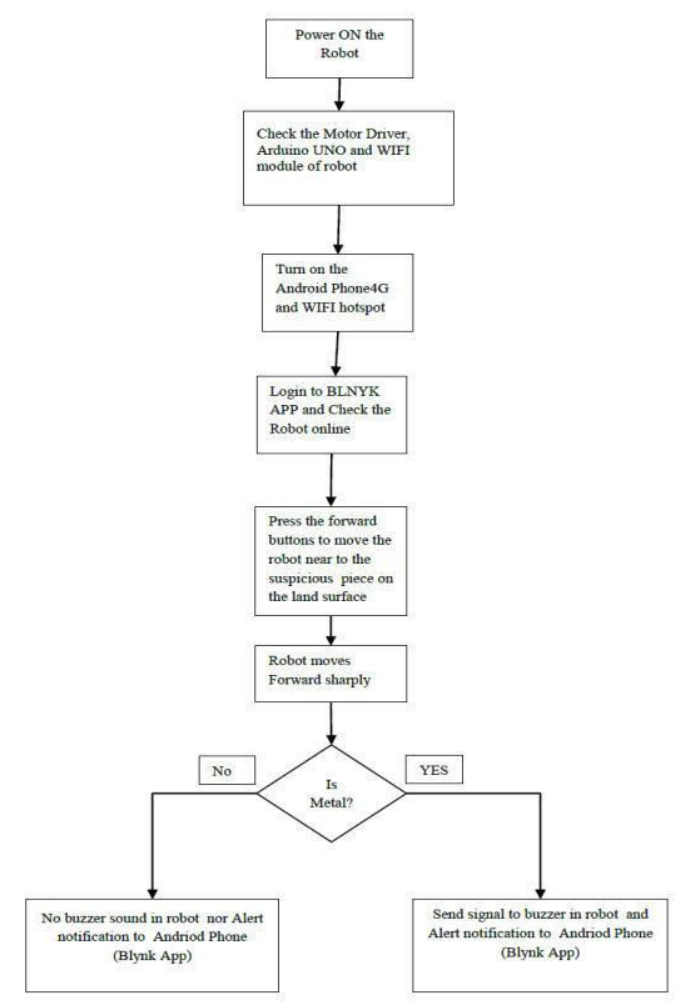

Figure 13. Motor Verification Flowchart

\section{Andriod Results And Discussion}

Following are the output results of multi-purpose field surveillance robot:

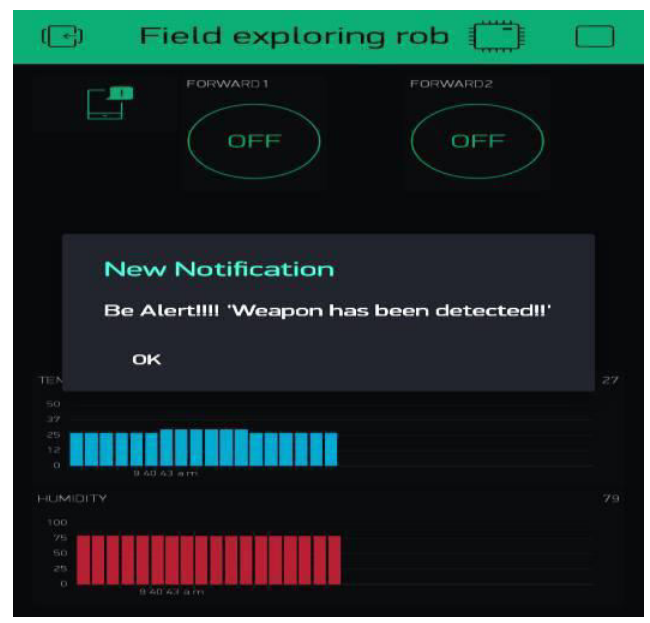

Figure 14. Metal Detection Verification screen 
The metal detector sensor correctly detects all kinds of ferrous objects which are metals, coins and any alloys of iron. These metals were detected up to a range of $10 \mathrm{~cm}$ depth from metal detector sensor mounted on the robot carrying vehicle. Usually landmines are buried on the land surface or within $10 \mathrm{~cm}$ of depth. Once power supply was switched on the robotic vehicle and WiFi hotspot was enabled on Android phone, the robot came online and connected to the Blynk App. Robot was driven through Android App and moved towards the suspicious item on the ground. Robot detected the suspicious item as metal and sent the alert message to Android App "Be Alert!!!! 'Weapon has been detected!!" .Thus the robot can identify metals and can be used for landmine detections.

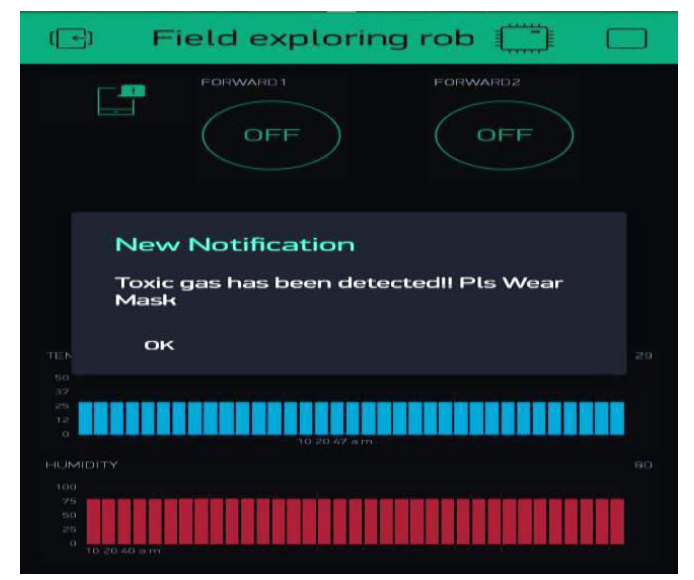

Figure 15. Gas Detection Verification screen

Resistance value of gas sensor is different to various kinds and various concentration gases. So, when using this component, sensitivity adjustment has been to the microcontroller to provide high output when the alcohol value of the sensor exceeds a threshold. The alcohol value was identified for smoke, LPG and was set on Arduino microcontroller. Power supply was switched on the robotic vehicle and WiFi hotspot was enabled on Android phone, the robot came online and connected to the Blynk App. Robot was driven through Android App and moved towards the gas area on the ground. Robot detected the toxic gas and sent the alert message to Android App "Toxic gas has been detected!! Pls Wear Mask". Thus the robot has successfully identified toxic gas and alerted the end user.

DHT11 measures the temperature using a thermistor and moisture using a moisture substrate electrode and negative temperature co-efficient thermistor. This sensor is on top of the robot vehicle to detect any anomalies in temperate and moisture reading of the area under surveillance. Power supply was switched on the robotic vehicle and WiFi hotspot was enabled on Android phone, the robot came online and connected to the Blynk App.Robot was driven through Android App and moved towards the area for the surveillance of temperature and moisture on the ground.

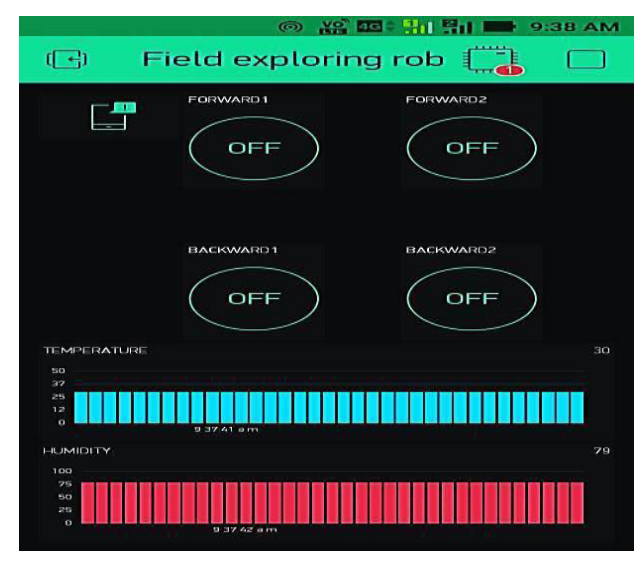

Figure 16. Temperature and Humidity Verification screen

Robot measured the Temperature and Moisture reading in the area and sent the values to the Blynk App. Thus the robot has successfully identified toxic gas and alerted the end user.

\section{Army Webpage Results And Discussion}

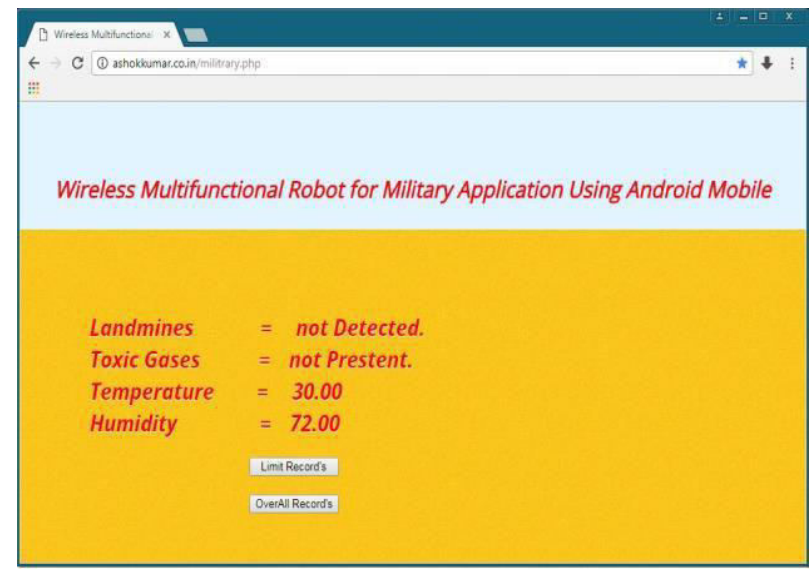

Figure 17. Army WebPage monitoring Section

Army webpage has designed for Cloud integration of robotic data and successfully host on the cloud servers. The webpage was designed using PHP, MySQL, JavaScript, HTML and JSP technologies and hosted on Cloud servers. All data are stored in secure database which can be viewed only at the Army Headquarters. This is a novel attempt to integrate multi-purpose field robot to dual cloud technologies.

\section{FUTURE WORK}

There is scope for improvement in future works like mounting a GPS module and wireless camera on the robot so tracking the location and controlling the movement of the robot will be easier from any remote location.By using GPS 
receiver, the user can know deploy the robot to any given location of the world using automatic command to the microcontroller. This will be path breaking improvement for robotic technology since the robot can do autonomous surveillance for fields without any man help. By fixing an IP camera, the robot can help user to control the robot seamlessly from remote location like army headquarters. The user will be able to remotely control the robot even when it goes out of sight.

\section{CONCLUSION}

Multi-purpose field surveillance robot has been successfully designed, constructed and integration tested using the best available resources. This multipurpose robot can be deployed in war fields for military use. The robots successfully detect metal and toxic gases so the robot instead of humans can be put to detect a dangerous item. Our robot prototype employs Wi-Fi technology hence it has wide range of operation and can cover better distance.

The robot is controlled by Android which is used by most popular mobile and available to any kind of people around the world. The robot is safely encoded with an authentication token which provides maximum security and hard for trafficking. The robot weighs less than a kilogram; hence it is easily portable and can be deployed on all kinds of terrain.

Integration of modern IoT technology has profoundly supplied bounteous information of the field area at anytime and anywhere in the world. Use of Cloud technology makes our robot a market demand product and a must for military operations.

\section{REFERENCES}

[1] M. Heidi, (1999) "Bluetooth technology and Implementations", John Wiley \& Sons.

[2] Piyare, R. and Tazil, M. (2011) "Bluetooth based home automation system using Android phones". IEEE 15TH International symposium on consumer electronics (ISCE), 1417 June 2011, Singapore.

[3] Potts, J. and Sukittanon, S. (2012) "Exploiting Bluetooth on android mobile devices for home security application", proceedings of southeastcan, 15-18 March 2012, Orlando, Florida, USA.

[4] "The Canadian Landmine Foundation - La Fondation des Mines Terrestres du Canada." Web. 18 Oct. 2009. .

[5] "How many land mines are in the world? Smart QandA: Answers and facts you can trust, verified with Encyclopedia.com." Web. 18 Oct. 2009. .
[6] "Landmines - the tragic statistics." Landmine Action Week, 20-26 May 2006. Web. 18 Oct. 2009.

[7] Cristina Turcu, Cornel Turcu and Vasile Gaitan, "Integrating robots into the Internet of Things", Issue 6, Volume 6, 2012, INTERNATIONAL JOURNAL OF CIRCUITS, SYSTEMS AND SIGNAL PROCESSING

[8] Tania Alauddin, Md. Tamzid Islam and Hasan U. Zaman, "Efficient Design of A Metal Detector Equipped RemoteControlled Robotic Vehicle", 978-1-4673-6621-2/16/2016 IEEE

[9] N.Saravanan , R.Kaviyarasi, P.Malini, "WIRELESS LAND MINE DETECTION AND SURVEILLANCE ROBOT" Volume 4, Issue 3, March -2017, International Journal of Advance Engineering and Research Development

[10] K.Vidyasagar, U.Nageshwarrao, K.Suresh and MD.Abdul Farooq; "Landmine detection robot using radio frequency communication"; JAIR volume 4, issue 2 july 2015 pp 51-55

[11] Bharat.J; "Automatic landmine detection and sweeper robot using microcontroller” volume 4, January 1,2015 IJMERR pp 485-492.

[12] "Robot controlled car using WI-FI module"; S.R. Madkar, vipul Mehta, NitinBhuvaniya, Maître parida; IJARCSSE volume 6 , issue 5 , may 2016.

[13] "Wireless control of surveillance robot"; kunalborker, Rohan gaikwad, Ajaysingh Rajput; IJARCSMS volume 2, issue 2, February 2014.

[14] W.M.M. Seada, E. Kamal and M. A. Koutb, "Wireless Robot for Personal Mine Detection and Extraction Equipped with a Wide Range Metal Detector Sensor", Volume 7, Issue 9, September-2016, International Journal of Scientific \& Engineering Research 